\title{
MicroRNA expression profiling studies on bronchopulmonary dysplasia: a systematic review and meta-analysis
}

\author{
Y. Yang, J. Qiu, Q. Kan, X.-G. Zhou and X.-Y. Zhou \\ Department of Neonates, \\ Nanjing Children's Hospital of Nanjing Medical University, Nanjing, China \\ Corresponding authors: X.Y. Zhou / X.G. Zhou \\ E-mail: yy860507@126.com / gzzhouxg@163.com
}

Genet. Mol. Res. 12 (4): 5195-5206 (2013)

Received February 26, 2013

Accepted July 18, 2013

Published October 30, 2013

DOI http://dx.doi.org/10.4238/2013.October.30.4

\begin{abstract}
Over the past several years, several microRNA (miRNA) expression profiling studies have been carried out on bronchopulmonary dysplasia (BPD) in mammalian lung tissues. The most effective way to identify these important miRNAs is to systematically search for similar signatures identified in multiple independent studies. Accordingly, a meta-analysis was conducted to review published miRNA expression profiling studies that compared miRNA expression profiles between BPD lung tissues and normal lung tissues. A vote-counting strategy that considered the total number of studies and time points reporting differential expression was applied. Furthermore, cut-off criteria of statistically significant differentially expressed miRNAs as defined by the author and their predicted target genes, if available, as well as the list of up- and down-regulated miRNA features, were collected and recorded. Results of the meta-analysis revealed that four up-regulated miRNAs (miRNA-21, miRNA-34a, miRNA-431, and Let-7f) and one down-regulated miRNA (miRNA-335) were differentially expressed in BPD lung tissues compared with normal groups. In addition, eight miRNAs (miRNA-146b, miRNA-29a, miRNA-503, miRNA-411,
\end{abstract}


miRNA-214, miRNA-130b, miRNA-382, and miRNA-181a-1*) were found to show differential expression not only in the process of normal lung development, but also during the progress of BPD. Finally, several meaningful target genes (such as the HPGD and NTRK genes) of common miRNAs (such as miRNA-21 and miRNA-141) were systematically predicted. These specific miRNAs may provide clues of the potential mechanisms involved in BPD. Further mechanistic and external validation studies are needed to confirm the clinical significance of these miRNAs in the development of BPD.

Key words: MicroRNA; Profiling; Bronchopulmonary dysplasia; Meta-analysis

\section{INTRODUCTION}

In the last few years, bronchopulmonary dysplasia (BPD) has become a common respiratory disease in preterm neonates, especially in those with severely reduced birth weights, such as very low birth weight infants and extremely low birth weight infants. Due to the effects of long-term oxygen therapy and assisted ventilation, many of these preterm infants consequently acquire different types of problems, such as highly reactive airway diseases, recurrent lower respiratory tract infections, feeding difficulties, and growth retardation. Currently, BPD is one of the most intractable clinical diseases in neonatal intensive care units, and is the main cause of infantile chronic respiratory diseases. Meanwhile, with the development of perinatal medicine, the incidence of BPD shows an upward trend following the improved survival rate for preterm babies. In China, its incidence has increased to $1.26 \%(156 / 12351$ neonates) according to a multicenter clinical study (Collaborative Study Group for Bronchopulmonary Dysplasia of Prematurity in China, 2011). Consequently, studies on BPD have received considerable attention by numerous researchers. However, despite ongoing research in this field, molecular understanding of this disease remains poor.

Increasing microRNA (miRNA) studies have emerged in the field of BPD. At the molecular level, miRNAs are a class of small, non-coding, highly stable RNAs that play important and complex roles in regulating mRNA and protein expression in various physiological and pathological processes, such as cellular differentiation, proliferation, angiogenesis, metabolism, and cancer development (Carrington and Ambros, 2003; Suárez and Sessa, 2009; Hatley et al., 2010). Therefore, microarray-based miRNA profiling assays have attracted increasing attention because they constitute an efficient methodology for parallel screening of the expression of hundreds of miRNAs using a high-throughput probe. With the aim of identifying meaningful miRNAs of BPD, over the past several years some investigators have carried out miRNAs expression profiling studies in mammalian lung tissues (Zhang et al., 2011; Dong et al., 2012; Bhaskaran et al., 2012). As a result, dozens of differentially expressed miRNAs have been identified. These miRNAs show both over- and under-expressed changes, depending on their target downstream genes. Given the large number of candidate signatures, a logical way to identify important miRNAs is to search for the intersection of the same signatures identified in multiple independent studies. The challenge is to effectively analyze and collect results of different miRNA expression profiling studies that used different profiling platforms 
and methods of evaluating differential expression levels, such as normalization or significance thresholds. To solve these difficulties, Chan et al. (2008) and Guan et al. (2012) proposed a vote-counting strategy to identify consistent markers when raw data are unavailable, which inspired the present meta-analysis of miRNA expression profiling studies in BPD.

The starting point of this systematic review and meta-analysis was to collect published miRNA expression profiling studies that compared miRNA expression profiles in BPD with those in control/normal groups. Then, the above-mentioned vote-counting strategy was used, which considered the total number of studies and time points reporting differential expression of specific miRNAs. We also ranked the predicted target genes of differentially expressed upregulated or down-regulated miRNAs, and investigated the possible biological processes and Kyoto Encyclopedia of Genes and Genomes (KEGG) pathways of these miRNAs.

\section{MATERIAL AND METHODS}

\section{Study selection}

PubMed and EMBASE were used to search for BPD miRNA expression profiling studies published from January 2003 to December 2012 (last accessed on December 15, 2012) using the following medical subject headings terms: 'bronchopulmonary dysplasia' and 'microRNAs' in combination with the key words 'profiling' or 'hyperoxia'. Eligible studies had to meet the following criteria: i) they were miRNA expression profiling studies in BPD; ii) they used lung tissue samples obtained from BPD animal models and corresponding control tissues for comparison; iii) they used miRNA microarray methods; iv) they reported cut-off criteria of differentially expressed miRNAs, and v) they reported the validation method set. Therefore, miRNA profiling studies using serum or sputum samples of BPD or lung epithelial cell lines, or those using different miRNA techniques were excluded. Review articles were also excluded.

\section{Data abstraction}

Two investigators (Y.Y. and J.Q.) independently evaluated and extracted the data using standard protocols, and all discrepancies were resolved by a third investigator (X.G.Z.). From the full text and corresponding supplementary information, the following eligibility items were collected and recorded for each study: author, journal and year of publication, location of study, methods and characteristics of animal modeling, platform of miRNA expression profiling, author defined cut-off criteria of statistically significant differentially expressed miRNAs and their predicted target genes (if available), and the list of up- and down-regulated miRNA features.

\section{Ranking}

Each included study comparing miRNA expression between BPD and normal/control lung tissues provided a list of differentially expressed miRNAs. Then, the following votecounting strategy, based on the method of ranking potential molecular biomarkers (Chan et al., 2008; Guan et al., 2012), was adopted in the meta-analysis. The differentially expressed miRNAs 
reported in each study were ranked according to order of importance based on the number of time points in studies that consistently reported the miRNA as differentially expressed and with a consistent direction of change. All comparisons were analyzed in a stepwise fashion using the online bioinformatic tool (http://jura.wi.mit.edu/bioc/tools/compare.php), and the ranking was performed with the Statistical Product and Service Solutions software (SPSS 13.0 for windows, SPSS Inc., Chicago, IL, USA).

\section{RESULTS AND DISCUSSION}

\section{Independent studies included}

A total of 106 relevant publications were indexed in PubMed and EMBASE. According to the inclusion criteria and identification of duplicate publications, only three independent studies (Zhang et al., 2011; Dong et al., 2012; Bhaskaran et al., 2012) were ultimately included in the analysis. The characteristics of these studies are listed in Table 1 in the order of publication date. Zhang et al. (2011) compared miRNA expression between BPD and control groups at three time points as postnatal day (P) 2, P7, and P21, which corresponds to just before, during, and after the lung septation. Furthermore, they also provided detailed accounts of miRNA expression during lung development in BPD. Dong et al. (2012) compared miRNA profiles at P14 and P29 between BPD and control groups. In addition, they listed possible target genes of the differentially expressed miRNAs using mRNA array analysis. Bhaskaran et al. (2012) used their own microarray platform to profile miRNAs in BPD rats at P13. They also presented the expression patterns of specific miRNAs during hyperoxia-mediated lung injury from embryonic day 16 to the adult stage. Furthermore, they performed a DNA microarray analysis to predict target genes of the identified miRNAs.

\section{Differentially expressed miRNAs between BPD and control groups}

A total of 89 differentially expressed miRNAs (including 77 up-regulated miRNAs and 12 down-regulated miRNAs) were reported in these three miRNA profiling studies that systematically compared BPD tissues with control tissues at seven different time points. Among the 77 up-regulated miRNAs, 30 miRNAs (38.9\%) were reported in at least two time points. All of the 30 up-regulated miRNAs showed a consistent direction. Four miRNAs (miRNA-21, miRNA-34a, miRNA-431, Let-7f) showed differential expression in at least two different studies. Among the 12 down-regulated miRNAs, only miRNA-335 (8.3\%) was differentially expressed in both time points reported in the two studies (the differentially up- or down-regulated miRNAs are listed in Tables 2 and 3).

The miRNAs identified above all play specific roles in lung homeostasis or lung development. For example, miRNA-21 has been mainly reported in lung cancer, and more than one study has demonstrated that its expression significantly increased in different types of lung cancer tissues (Solomides et al., 2012; Donnem et al., 2012). Zhong et al. (2012) and Liu et al. (2013) further reported that miRNA-21 increased cell growth, promoted migration and invasion, and enhanced chemo- or radio-resistance of non-small cell lung cancer cells, mainly by acting on the phosphatase and tensin homolog signaling pathway and by down-regulating human mutS homolog 2 , one of the core mismatch repair genes. In addi- 


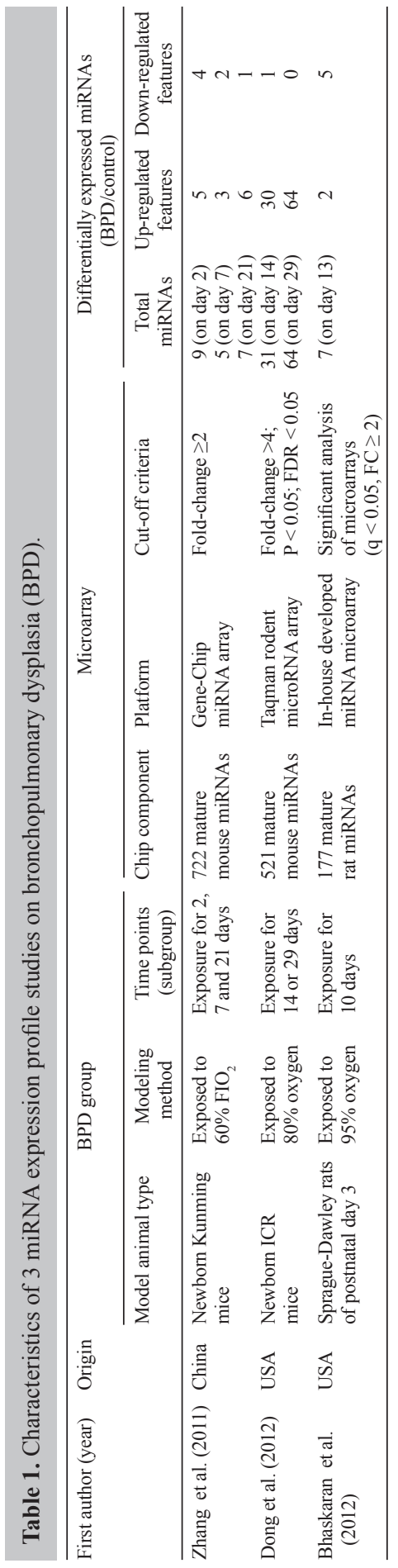


tion, Vaporidi et al. (2012) reported that miRNA-21 could be highly up-regulated by high tidal volume ventilation, and could exert a negative effect on lung compliance through increasing alveolar-arterial oxygen differences and the level of inflammation of the associated protein. Yang et al. (2012a) further showed that miRNA-21 plays an important role in the pathogenesis of chronic hypoxia-induced pulmonary vascular remodeling. Over-expression of miRNA-21 was shown to enhance the proliferation of human pulmonary artery smooth muscle cells (PASMCs) in vitro and increased the expression of cell proliferation-associated proteins, such as proliferating cell nuclear antigen, cyclin D1, and Bcl-xL. Together, this study suggests that these specific miRNAs might also participate in the pathological process of BPD through similar mechanisms.

\section{Differentially expressed miRNAs during lung development with BPD}

Zhang et al. (2011) screened 36 specific microRNAs that showed aberrant regulation in the lung development of BPD mice only, and Dong et al. (2012) showed an intersection of dynamically regulated miRNAs in normal lung alveolar development and hyperoxia-induced BPD. To ensure accuracy and comparability of these two studies, we also investigated the intersection for the miRNA sets between normal lung development and BPD lung tissues based on original data of Zhang et al. (2011). Finally, based on data of these two references, we found eight miRNAs (miRNA-146b, miRNA-29a, miRNA-503, miRNA-411, miRNA-214, miRNA-130b, miRNA-382, and miRNA-181a-1*) showing differential expression not only in normal lung development, but also in the progress of BPD (Table 4). Previous research has revealed that miRNA-146b is mainly involved in innate immune and inflammation responses (Taganov et al., 2006; Perry et al., 2009). It has also been demonstrated to play a central role in the negative feedback regulation of interleukin- $1 \beta$-induced inflammation by down-regulating two proteins, IRAK1 and TRAF6, involved in Toll/interleukin-1 receptor signaling (Taganov et al., 2006; Perry et al., 2008). In addition, our research group previously demonstrated that miRNA-146b also showed higher expression levels in normal lung development (Yang et al., 2012b). Therefore, miRNA-146b could play an important role in regulating the BPD immune response in the progress of inflammation. These identified miRNAs might also be essential to the pathogenesis of BPD.

\section{Predicted target genes of differentially expressed miRNAs}

Both Dong et al. (2012) and Bhaskaran et al. (2012) predicted the possible target genes of the identified miRNAs. The latter performed a DNA microarray analysis to detect mRNA changes using the same samples for miRNA microarray analysis. To avoid false-positive and false-negative results, they further obtained intersection elements between the DNA microarray set and the TargetScan/miRnada database. Based on the basic regulating mechanism of miRNA, at the post-transcription level, down-regulated miRNAs generally leads to up-regulation in expression of targeted genes and vice versa. Similarly, Dong et al. (2012) also used an mRNA array to profile the expression of target genes, and then performed a Gene Ontology analysis to determine the biological process of BPD. In order to identify target genes of differentially expressed miRNAs, an intersection of two databases (miRBase and TargetScan) was also conducted in their study (Tables 5 and 6). 
Table 2. Differentially up-regulated miRNAs at seven time points in the bronchopulmonary dysplasia (BPD) group.

\begin{tabular}{|c|c|c|c|c|c|c|}
\hline $\mathrm{P} 2 *$ & $\mathrm{P} 7 *$ & P13* & P14* & $\mathrm{P} 21 *$ & $\mathrm{P} 29 *$ & Count \\
\hline- & - & - & - & - & miRNA-15a & 1 \\
\hline miRNA-15b & - & - & - & - & - & 1 \\
\hline miRNA-20b & - & - & - & - & - & 1 \\
\hline- & - & miRNA-21 & miRNA-21 & - & miRNA-21 & 3 \\
\hline - & - & - & miRNA-22 & - & miRNA-22 & 2 \\
\hline- & - & - & - & - & miRNA-22* & 1 \\
\hline - & - & - & miRNA-27a & - & miRNA-27a & 2 \\
\hline - & - & - & - & - & miRNA-27b & 1 \\
\hline - & - & - & miRNA-29a & - & miRNA-29a & 2 \\
\hline - & - & - & miRNA-29c & - & miRNA-29c & 2 \\
\hline - & - & - & - & - & miRNA-30d & 1 \\
\hline- & miRNA-30e & - & - & - & - & 1 \\
\hline- & - & - & - & - & $\operatorname{miRNA}-31^{*}$ & 1 \\
\hline- & - & miRNA-34a & miRNA-34a & - & miRNA-34a & 3 \\
\hline - & - & - & miRNA-34c & - & miRNA-34c & 2 \\
\hline - & - & - & - & - & miRNA-99a & 1 \\
\hline - & - & - & - & - & miRNA-100 & 1 \\
\hline- & - & - & miRNA-101b & - & miRNA-101b & 2 \\
\hline- & - & - & miRNA-103 & - & miRNA-103 & 2 \\
\hline miRNA-106a & - & - & - & - & - & 1 \\
\hline- & - & - & - & - & miRNA-106b & 1 \\
\hline- & miRNA-122 & - & - & - & - & 1 \\
\hline- & - & - & miRNA-124 & - & miRNA-124 & 2 \\
\hline miRNA-128 & - & - & - & - & - & 1 \\
\hline- & - & - & miRNA-130a & - & miRNA-130a & 2 \\
\hline- & - & - & miRNA-130b & - & miRNA-130b & 2 \\
\hline- & - & - & - & miRNA-133 & - & 1 \\
\hline - & - & - & - & - & miRNA-135b & 1 \\
\hline- & - & - & miRNA-141 & - & miRNA-141 & 2 \\
\hline- & - & - & - & - & miRNA-142-3p & 1 \\
\hline - & - & - & miRNA-143 & - & miRNA-143 & 2 \\
\hline- & - & - & - & - & miRNA-148a & 1 \\
\hline- & - & - & - & - & miRNA-152 & 1 \\
\hline- & - & - & - & - & miRNA-181a & 1 \\
\hline- & - & - & miRNA-181c & - & miRNA-181c & 2 \\
\hline- & - & - & - & - & miRNA-199a-3p & 1 \\
\hline - & - & - & - & miRNA-205 & - & 1 \\
\hline - & - & - & - & - & miRNA-210 & 1 \\
\hline- & - & - & miRNA-214 & - & miRNA-214 & 2 \\
\hline- & - & - & - & - & miRNA-218 & 1 \\
\hline- & - & - & - & - & miRNA-221 & 1 \\
\hline- & - & - & miRNA-301a & - & miRNA-301a & 2 \\
\hline- & - & - & miRNA-301b & - & miRNA-301b & 2 \\
\hline- & - & - & - & - & miRNA-322 & 1 \\
\hline - & - & - & - & - & miRNA-340-5p & 1 \\
\hline- & miRNA-365-5p & - & - & - & - & 1 \\
\hline- & - & - & - & - & miRNA-378 & 1 \\
\hline- & - & - & - & miRNA-379 & - & 1 \\
\hline- & - & - & miRNA-382 & - & miRNA-382 & 2 \\
\hline- & - & - & - & - & miRNA-411 & 1 \\
\hline - & - & - & miRNA-431 & miRNA-431 & miRNA-431 & 3 \\
\hline - & - & - & - & miRNA-449a & - & 1 \\
\hline- & - & - & miRNA-451 & - & miRNA-451 & 2 \\
\hline - & - & - & - & - & miRNA-455* & 1 \\
\hline- & - & - & miRNA-494 & - & miRNA-494 & 2 \\
\hline- & - & - & - & - & miRNA-497 & 1 \\
\hline- & - & - & miRNA-503 & - & miRNA-503 & 2 \\
\hline - & - & - & - & - & miRNA-532-5b & 1 \\
\hline - & - & - & miRNA-652 & - & miRNA-652 & 2 \\
\hline - & - & - & miRNA-672 & - & miRNA-672 & 2 \\
\hline - & - & - & miRNA-680 & - & - & 1 \\
\hline- & - & - & miRNA-685 & - & miRNA-685 & 2 \\
\hline
\end{tabular}

Continued on next page 


\begin{tabular}{|c|c|c|c|c|c|c|}
\hline $\mathrm{P} 2 *$ & $\mathrm{P} 7 *$ & $\mathrm{P} 13^{*}$ & P14* & $\mathrm{P} 21^{*}$ & $\mathrm{P} 29^{*}$ & Count \\
\hline- & - & - & - & - & miRNA-690 & 1 \\
\hline - & - & - & - & - & miRNA-699 & 1 \\
\hline- & - & - & miRNA-706 & - & miRNA-706 & 2 \\
\hline- & - & - & - & - & miRNA-709 & 1 \\
\hline- & - & - & - & - & miRNA-744 & 1 \\
\hline- & - & - & miRNA-801 & - & miRNA-801 & 2 \\
\hline- & - & - & - & - & miRNA-805 & 1 \\
\hline miRNA-883b-3p & - & - & - & - & - & 1 \\
\hline- & - & - & - & - & miRNA-let-7a & 1 \\
\hline - & - & - & - & - & miRNA- let-7b & 1 \\
\hline- & - & - & miRNA- let-7c & - & miRNA- let-7c & 2 \\
\hline- & - & - & - & - & miRNA- let-7d & 1 \\
\hline- & - & - & - & - & miRNA- let-7e & 1 \\
\hline- & - & - & - & miRNA-let-7f & miRNA- let-7f & 2 \\
\hline - & - & - & - & - & miRNA-let-7i & 1 \\
\hline
\end{tabular}

Editied based on the three profiling studies on BPD. *Postnatal day (P2), P7 and P21 represent the time points reported in Zhang et al. (2011); P13 represents the time point reported in Dong et al. (2012); P14 and P29 represent the time points reported in Bhaskaran et al. (2012).

\begin{tabular}{|c|c|c|c|c|c|c|}
\hline $\mathrm{P} 2 *$ & $\mathrm{P} 7 *$ & P13* & P14* & $\mathrm{P} 21^{*}$ & P29* & Count \\
\hline \multirow{3}{*}{ miRNA-122 } & - & - & miRNA-10b & - & - & 1 \\
\hline & - & - & - & - & - & 1 \\
\hline & - & miRNA-126* & - & - & - & 1 \\
\hline \multirow{3}{*}{ miRNA-139p-3p } & - & - & - & - & - & 1 \\
\hline & - & miRNA-150 & - & - & - & 1 \\
\hline & - & miRNA-151* & - & - & - & 1 \\
\hline miRNA-299 & - & - & - & - & - & 1 \\
\hline \multirow{5}{*}{ miRNA-300* } & - & - & - & - & - & 1 \\
\hline & miRNA-335-5p & miRNA-335 & - & - & - & 2 \\
\hline & - & miRNA-342 & - & - & - & 1 \\
\hline & miRNA-714 & - & - & - & - & 1 \\
\hline & - & - & - & miRNA-720 & - & 1 \\
\hline
\end{tabular}

Editied based on the three profiling studies on BPD. *Postnatal day (P2), P7 and P21 represent the time points reported in Zhang et al. (2011); P13 represents the time point reported in Dong et al. (2012); P14 and P29 represent the time points reported in Bhaskaran et al. (2012).

Two miRNAs (miRNA-21 and miRNA-141) were predicted in BPD by both Dong et al. (2012) and Bhaskaran et al. (2012). Although the predicted target genes were different (Table 5) in the two studies, these targets might participate in the development of BPD. For example, 15-hydroxyprostaglandin dehydrogenase (15-PGDH, HPGD), which is involved in the initial inactivation of prostaglandins (PGs), is mediated by HPGD, and PGs are potent mediators of several biological processes, including inflammation and reproduction (Aisemberg et al., 2012). In BPD, miRNA-21 and miRNA-141 might also augment PG-E synthase activity to promote inflammation by decreasing HPGD protein expression. Therefore, some predicted genes do show characteristics of being targets. A similar example was provided in Sciesielski et al. (2009) study, which revealed that hypoxia could stimulate the expression of the tyrosine kinase B (TrkB) neurotrophin (NTRK) receptor in the airway epithelium. Activation of TrkB signaling could enhance mechanical airway contractility to acetylcholine. Furthermore, Kwapiszewska et al. (2012) identified the BDNF-TrkB-ERK1/2 pathway as a 
proliferative signaling pathway for PASMC in pulmonary hypertension. This pathway could augment smooth muscle cell proliferation in pulmonary hypertension. Because interference with this pathway may serve as an attractive reverse remodeling approach, it may be helpful to explain airway remodeling in BPD.

Table 4. Differentially expressed miRNAs during lung development with bronchopulmonary dysplasia (BPD).

\begin{tabular}{|c|c|c|c|c|c|}
\hline Zhang et al. (2011) & Dong et al. (2012) & Zhang et al. (2011) & Dong et al. (2012) & Zhang et al. (2011) & Dong et al. (2012) \\
\hline miRNA-146b & $m i R N A-146 b$ & miRNA-126-3p & NR & NR & let- $7 \mathrm{~b}$ \\
\hline$m i R N A-29 a$ & $m i R N A-29 a$ & miRNA-127 & NR & NR & let-7c \\
\hline miRNA-503 & $m i R N A-503$ & miRNA-134 & NR & NR & let-7d \\
\hline$m i R N A-411$ & $m i R N A-411$ & miRNA-434-3p & NR & NR & let-7e \\
\hline$m i R N A-214$ & $\operatorname{miRNA}-214$ & miRNA-187 & NR & NR & let-7i \\
\hline miRNA-130b & miRNA-130b & miRNA-668 & NR & NR & miRNA-135b \\
\hline miRNA-382 & miRNA-382 & miRNA-337-5p & NR & NR & miRNA-141 \\
\hline miRNA-181a-1* & miRNA-181a-1* & miRNA-487b & NR & NR & miRNA-142-3p \\
\hline miRNA-125b-5p & $\mathrm{NR}^{*}$ & miRNA-122 & NR & NR & miRNA-21 \\
\hline miRNA-30b & NR & miRNA-434-5p & NR & NR & miRNA-210 \\
\hline miRNA-146a & NR & miRNA-127* & NR & NR & miRNA-27a \\
\hline miRNA-31 & NR & miRNA-300 & NR & NR & miRNA-29c \\
\hline miRNA-497 & NR & miRNA-665 & NR & NR & miRNA-301a \\
\hline miRNA-99a & NR & miRNA-449a & NR & NR & miRNA-301b \\
\hline miRNA-17 & NR & miRNA-466f-3p & NR & NR & miRNA-322* \\
\hline miRNA-18a & NR & miRNA-409-5p & NR & NR & miRNA-431 \\
\hline miRNA-298 & NR & miRNA-541 & NR & NR & miRNA-451 \\
\hline miRNA-296-3p & NR & miRNA-329 & NR & NR & miRNA-494 \\
\hline miRNA-351 & NR & miRNA-466f-5p & NR & NR & miRNA-532-5p \\
\hline miRNA-30e & NR & miRNA-342-3p & NR & NR & miRNA-672 \\
\hline miRNA-100 & NR & miRNA-342-5p & NR & NR & miRNA-690 \\
\hline \multirow[t]{2}{*}{ miRNA-409-3p } & NR & miRNA-24-2* & NR & NR & miRNA-706 \\
\hline & & miRNA-708 & NR & NR & miRNA-709 \\
\hline
\end{tabular}

$\mathrm{NR}=$ not reported. Eight miRNAs (in italics) showing differential expression not only in normal lung development but also in the progress of BPD.

\section{Predicted biological processes and KEGG pathways}

Although all three of these articles described the expression profile of miRNAs in BPD, they did not systematically predict the biological process and pathway of the identified miRNAs. Therefore, we used BINGO 2.1 (http://www.psb.ugent.be/cbd/papers/BiNGO/ Home.html) to predict the biological process of all miRNAs that were identified as differentially expressed in the three references. We further applied the Database for Annotation, Visualization and Integrated Discovery (DAVID, National Institutes of Health) (http://david. abcc.ncifcrf.gov/), which can provide relatively accurate and systematic pathways of BPD (Table S1).

With respect to miRNA-34a, the biological process predicted by BINGO showed that it could participate in several pathways that are associated with BPD, such as multicellular organismal development and system developmental processes. Other miRNAs, such as miRNA-141 and miRNA-21, also appear to play possible roles in the regulation of biological, metabolic, and cellular processes, which may also be involved in the pathogenesis of BPD. However, because most of these target genes have not yet been confirmed by further experiments, these functions remain purely speculative for the time being, and will not be discussed in more detail here. 
Table 5. Predicted down-regulated target genes of differentially expressed up-regulated miRNAs.

\begin{tabular}{|c|c|c|c|c|}
\hline \multirow[b]{2}{*}{ Name } & \multicolumn{2}{|c|}{ Bhaskaran et al. (2012) } & \multicolumn{2}{|c|}{ Dong et al. (2012) } \\
\hline & TargetScan & miRnada & miRBase and TargetScan (intersection P14) & miRBase and TargetScan (intersection P29) \\
\hline miRNA-21 & HPGD & HPGD & RTN4; VCL & RTN4; VCL \\
\hline miRNA-34a & NR & NR & EHMT2; LSP1 & EHMT2; LSP1 \\
\hline miRNA-141 & HPGD & HPGD & CNTN1; NTRK2 & CNTN1; NTRK2 \\
\hline miRNA-27b & NR & NR & NR & HILPDA; DIl4; LAMC2; PSEN1 \\
\hline miRNA-214 & NR & NR & ACTN1; CHI3L3; LSP1; NGP & ACTN1; CHI3L3; LSP; NGP \\
\hline miRNA-378 & NR & NR & NR & ADIPOQ; ANPEP; HVCN1; LAT; LEFL; SCARB1 \\
\hline miRNA-106b & NR & NR & NR & AFF2; CCNG2; FABP4; RASIP1; RORA \\
\hline miRNA-142-3p & NR & NR & NR & AFF2; FABP4; GJA4; TGM2 \\
\hline miRNA-301a & NR & NR & AGTR1A; GAP43; NTRK2 & AGTR1A; ATRX; EAR2; GAP43; NTRK2 \\
\hline miRNA-301b & NR & NR & AGTR1A; GAP43; ATRX; NTRK2; S1PR3 & AGTR1A; ATRX; EAR2; Gap43; NTRK2; S1PR3 \\
\hline miRNA-451 & NR & NR & $\begin{array}{l}\text { AGTR1A; CAP1; CHI3L3; } \\
\text { MS4A1; S1PR1; YES1 }\end{array}$ & $\begin{array}{l}\text { AGTR1A; CAP1; CHI3L3; } \\
\text { MS4A1; S1PR1; YES1 }\end{array}$ \\
\hline miRNA-709 & NR & NR & NR & $\begin{array}{l}\text { ALAS2; BIRC5; CD97; DST; LCK; } \\
\text { PLUNC; TAPBP; TNXB }\end{array}$ \\
\hline miRNA-135b & NR & NR & NR & ANG; LAT \\
\hline miRNA-210 & NR & NR & NR & $\begin{array}{l}\text { ANG; ANG3; CXCR4; LEF1; MAN2B1; } \\
\text { RORA; SIGLECG }\end{array}$ \\
\hline miRNA-699 & NR & NR & NR & ANG3; CD97; H2-Q7; HVCN1; LCK \\
\hline Let-7b & NR & NR & NR & ANPEP; BIRC5; DOCK2; HSPB1; IFI47; PLUNC \\
\hline Let-7d & NR & NR & NR & ANPEP; BIRC5; DOCK2; GJA4; PLUNC \\
\hline Let-7e & NR & NR & NR & ANPEP; BIRC5; IFI47 \\
\hline Let-7f & NR & NR & NR & ANPEP; BIRC5; DOCK2; PLUNC \\
\hline Let-7i & NR & NR & NR & ANPEP; BIRC5; ITGAX; PECAM1; PLUNC \\
\hline miRNA-101b & NR & NR & ATRX; GJA1; NPNT & ATRX; GJA1; NPNT \\
\hline miRNA-103 & NR & NR & AXL; ITGB2 & AXL; ITGB2 \\
\hline miRNA-27a & NR & NR & BCAM; CDH5; GAP43; NGP & BCAM; CDH5; GAP43; NGP \\
\hline miRNA-218 & NR & NR & NR & BIRC5; CD9; CYTIP \\
\hline miRNA-221 & NR & NR & NR & BIRC5; HDAC7; SERPINF1; SQSTM1 \\
\hline miRNA-22* & NR & NR & NR & BRE; DOCK2; H2-D1; ICAM2; IL16 \\
\hline miRNA-744 & NR & NR & NR & BRE; EMILIN2; GJA4; H2-Q7 \\
\hline miRNA-22 & NR & NR & CAP1; CHI3L3 & CAP1; CHI3L3 \\
\hline miRNA-382 & NR & NR & CAP1; CNTN1; ETS1 & CAP1; CNTN1; ETS1 \\
\hline miRNA-100 & NR & NR & NR & CCNG2; LCK; RORA; TNXB \\
\hline miRNA-340-5p & NR & NR & NR & $\mathrm{CCNG} 2 ; \mathrm{CD} 9$ \\
\hline miRNA-99a & NR & NR & NR & CCNG2; ITGA3; LAMC2; LCK; RORA; TNXB \\
\hline miRNA-322 & NR & NR & NR & CD97; PECAM1; PLUNC \\
\hline miRNA-497 & NR & NR & NR & CD97; DOCK2; PECAM1; PLUNC; RASSF5 \\
\hline miRNA-532-5p & NR & NR & NR & CFD; GJA4 \\
\hline miRNA-124 & NR & NR & CNTN1; F7 & CNTN1; F7 \\
\hline miRNA-672 & NR & NR & CSF3R TRP53 & CSF3R; TRP53 \\
\hline miRNA-690 & NR & NR & NR & CTNNB1; DOCK2; HCFC1; PRKCE; SELPLG \\
\hline miRNA-30d & NR & NR & NR & DLL4; PRKCE; PTPRC \\
\hline $\operatorname{miRNA}-455^{*}$ & NR & NR & NR & DOCK2; SQSTM1 \\
\hline miRNA-148a & NR & NR & NR & $\begin{array}{l}\text { DST; ENPP2; H2-D1; H2-Q7; ICAM2; } \\
\text { OMD; SIGLECG }\end{array}$ \\
\hline miRNA-199a-3p & NR & NR & NR & DST; FERMT3; GJA4; HSPB1; LAMC2 \\
\hline miRNA-805 & NR & NR & NR & DST; ENPP2; IFI47; IL1B \\
\hline miRNA-34c & NR & NR & EHMT2 & EHMT2 \\
\hline miRNA-706 & NR & NR & EHMT2; EDNRA; RAC2 & EHMT2; EDNRA; RAC2 \\
\hline miRNA-152 & NR & NR & NR & ENPP2; H2-D1; H2-Q7; SIGLECG \\
\hline miRNA-181c & NR & NR & F7; FGFR2 & F7; FGFR2 \\
\hline miRNA-29a & NR & NR & GAB1; PIK3R1 & GAB1; PIK3R1 \\
\hline miRNA-29c & NR & NR & GAB1; NTRK2; PIK3R1 & Gab1; Ntrk2; Pik3r1 \\
\hline miRNA--130a & NR & NR & GAP43; S1PR1 & GAP43; S1PR1 \\
\hline miRNA-130b & NR & NR & GAP43; S1PR1 & GAP43; S1PR1 \\
\hline miRNA-181a & NR & NR & NR & H2-D1; HPGD; LAMC2; SEMA6A; SQSTM1 \\
\hline miRNA-15a & NR & NR & NR & HSPB1; PLUNC; SORBS1 \\
\hline miRNA-685 & NR & NR & ITGB2; RTN4 & ITGB2; RTN4 \\
\hline miRNA-411 & NR & NR & NR & LEF1 \\
\hline miRNA-494 & NR & NR & MAPK6 & MAPK6 \\
\hline miRNA-503 & NR & NR & MKNK1; TXNIP & MKNK1; TXNIP \\
\hline miRNA-143 & NR & NR & PXN; VTN & PXN; VTN \\
\hline miRNA-652 & NR & NR & SIRPA & SIRPA \\
\hline miRNA-31* & NR & NR & NR & SYT7 \\
\hline
\end{tabular}

$\mathrm{NR}=$ not reported. ${ }^{\text {BBecause of two different time points (P14 and P29) described in their original article, we }}$ defined two groups here: one is the intersection of target genes at P14 based on miRBase and TargetScan, and the other is the intersection of target genes at P29. 
Table 6. Predicted up-regulated target genes of differentially expressed down-regulated miRNAs.

\begin{tabular}{|c|c|c|c|c|}
\hline \multirow[b]{2}{*}{ Name } & \multicolumn{2}{|c|}{ Bhaskaran et al. (2012) } & \multicolumn{2}{|c|}{ Dong et al. (2012) } \\
\hline & TargetScan & miRnada & $\begin{array}{l}\text { miRBase and TargetScan } \\
\quad \text { (intersection P14) }\end{array}$ & $\begin{array}{l}\text { miRBase and TargetScan } \\
\quad(\text { intersection P29) }\end{array}$ \\
\hline miRNA-150 & $\begin{array}{l}\text { BASP1; CTGF; EBF1; } \\
\text { IGF1; GPNMB }\end{array}$ & CTGF; Fetub; DES; GPNMB & NR & NR \\
\hline miRNA-335 & PGK1; PRKAA2; ATP1B1 & SOD2; PRKAA2; ATP1B1 & NR & NR \\
\hline $\operatorname{miRNA}-126^{*}$ & / & ATP1B1; Clca2; Vim & NR & NR \\
\hline miRNA-151* & IGF1; PRKAA2 & IGF1; Retnla & NR & NR \\
\hline miRNA-342-3p & PRKAA2; S100 g; SOD2 & $\begin{array}{r}\text { GPNMB; LDHA; } \\
\text { S100a10; SOD2 }\end{array}$ & NR & NR \\
\hline miRNA-342-5p & CTGF; DES; EBF1; SP4 & CTGF; Des; Vim & NR & NR \\
\hline miRNA-10b & NR & NR & NR & NR \\
\hline
\end{tabular}

$\mathrm{NR}=$ not reported. "Because of two different time points (P14 and P29) described in their original article, we defined two groups here: one is the intersection of target genes at P14 based on miRBase and TargetScan, and the other is the intersection of target genes at P29.

\section{CONCLUSION}

In summary, through this meta-analysis and systematic review, four differentially expressed up-regulated miRNAs (miRNA-21, miRNA-34a, miRNA-431, and Let-7f) and one differentially expressed down-regulated miRNA (miRNA-335) were found in BPD lung tissues compared with normal groups. These specific miRNAs may also provide some clues of the potential biomarkers in BPD. Furthermore, eight miRNAs (miRNA-146b, miRNA-29a, miRNA-503, miRNA-411, miRNA-214, miRNA-130b, miRNA-382, and miRNA-181a-1*) were found to show differential expression not only in normal lung development, but also during the progress of BPD. Finally, several meaningful target genes (such as the HPGD and NTRK genes) of common miRNAs (such as miRNA-21 and miRNA-141) were systematically predicted. These specific miRNAs may play potential roles in BPD. Further mechanistic and external validation studies are clearly needed to confirm the clinical significance and role of the identified miRNAs in the progress of BPD.

\section{ACKNOWLEDGMENTS}

Research supported by grants from the Project Foundation of Jiangsu Province Health Department (\#H200642).

\section{Conflicts of interest}

The authors declare no conflict of interest.

\section{Supplementary material}

\section{REFERENCES}

Aisemberg J, Bariani MV, Vercelli CA, Wolfson ML, et al. (2012). Lipopolysaccharide-induced murine embryonic resorption involves nitric oxide-mediated inhibition of the $\mathrm{NAD}^{+}$-dependent 15-hydroxyprostaglandin dehydrogenase. Reproduction 144: 447-454. 
Bhaskaran M, Xi D, Wang Y, Huang C, et al. (2012). Identification of microRNAs changed in the neonatal lungs in response to hyperoxia exposure. Physiol. Genomics 44: 970-980.

Carrington JC and Ambros V (2003). Role of microRNAs in plant and animal development. Science 301: 336-338.

Chan SK, Griffith OL, Tai IT and Jones SJ (2008). Meta-analysis of colorectal cancer gene expression profiling studies identifies consistently reported candidate biomarkers. Cancer Epidemiol. Biomarkers Prev. 17: 543-552.

Collaborative Study Group for Bronchopulmonary Dysplasia of Prematurity in China (2011). Incidence and risk factors of bronchopulmonary dysplasia in premature infants in 10 hospitals in China. Chin. J. Pediatr. 49: 655-662.

Dong J, Carey WA, Abel S, Collura C, et al. (2012). MicroRNA-mRNA interactions in a murine model of hyperoxiainduced bronchopulmonary dysplasia. BMC Genomics 13: 204.

Donnem T, Fenton CG, Lonvik K, Berg T, et al. (2012). MicroRNA signatures in tumor tissue related to angiogenesis in non-small cell lung cancer. PLoS One 7: e29671.

Guan P, Yin Z, Li X, Wu W, et al. (2012). Meta-analysis of human lung cancer microRNA expression profiling studies comparing cancer tissues with normal tissues. J. Exp. Clin. Cancer Res. 31: 54.

Hatley ME, Patrick DM, Garcia MR, Richardson JA, et al. (2010). Modulation of K-Ras-dependent lung tumorigenesis by MicroRNA-21. Cancer Cell 18: 282-293.

Kwapiszewska G, Chwalek K, Marsh LM, Wygrecka M, et al. (2012). BDNF/TrkB signaling augments smooth muscle cell proliferation in pulmonary hypertension. Am. J. Pathol. 181: 2018-2029.

Liu ZL, Wang H, Liu J and Wang ZX (2013). MicroRNA-21 (miR-21) expression promotes growth, metastasis, and chemo- or radioresistance in non-small cell lung cancer cells by targeting PTEN. Mol. Cell Biochem. 372: 35-45.

Perry MM, Moschos SA, Williams AE, Shepherd NJ, et al. (2008). Rapid changes in microRNA-146a expression negatively regulate the IL-1beta-induced inflammatory response in human lung alveolar epithelial cells. J. Immunol. 180: 5689-5698.

Perry MM, Williams AE, Tsitsiou E, Larner-Svensson HM, et al. (2009). Divergent intracellular pathways regulate interleukin-1beta-induced miR-146a and miR-146b expression and chemokine release in human alveolar epithelial cells. FEBS Lett. 583: 3349-3355.

Sciesielski LK, Paliege A, Martinka P and Scholz H (2009). Enhanced pulmonary expression of the TrkB neurotrophin receptor in hypoxic rats is associated with increased acetylcholine-induced airway contractility. Acta Physiol. 197: 253-264.

Solomides CC, Evans BJ, Navenot JM, Vadigepalli R, et al. (2012). MicroRNA profiling in lung cancer reveals new molecular markers for diagnosis. Acta Cytol. 56: 645-654.

Suárez Y and Sessa WC (2009). MicroRNAs as novel regulators of angiogenesis. Circ. Res. 104: 442-454.

Taganov KD, Boldin MP, Chang KJ and Baltimore D (2006). NF-kappaB-dependent induction of microRNA miR-146, an inhibitor targeted to signaling proteins of innate immune responses. Proc. Natl. Acad. Sci. U. S. A. 103: 12481-12486.

Vaporidi K, Vergadi E, Kaniaris E, Hatziapostolou M, et al. (2012). Pulmonary microRNA profiling in a mouse model of ventilator-induced lung injury. Am. J. Physiol. Lung Cell Mol. Physiol. 303: L199-L207.

Yang S, Banerjee S, Freitas A, Cui H, et al. (2012a). miR-21 regulates chronic hypoxia-induced pulmonary vascular remodeling. Am. J. Physiol. Lung Cell Mol. Physiol. 302: L521-L529.

Yang Y, Kai G, Pu XD, Qing K, et al. (2012b). Expression profile of microRNAs in fetal lung development of SpragueDawley rats. Int. J. Mol. Med. 29: 393-402.

Zhang X, Peng W, Zhang S, Wang C, et al. (2011). MicroRNA expression profile in hyperoxia-exposed newborn mice during the development of bronchopulmonary dysplasia. Respir. Care 56: 1009-1015.

Zhong Z, Dong Z, Yang L and Gong Z (2012). miR-21 induces cell cycle at S phase and modulates cell proliferation by down-regulating hMSH2 in lung cancer. J. Cancer Res. Clin. Oncol. 138: 1781-1788. 